\title{
El papel de la sílaba en la codificación posicional de las representaciones ortográficas
}

\author{
Ascensión Pagán ${ }^{1,2 *}$, Javier Marín ${ }^{1}$ y Manuel Perea ${ }^{3}$ \\ ${ }^{1}$ Universidad de Murcia (España) \\ ${ }^{2}$ Universidad de Southampton (Reino Unido) \\ ${ }^{3}$ Universitat de València (España)
}

\begin{abstract}
Resumen: El objetivo de este trabajo consiste en estudiar si la posición de las letras se codifica de una forma absoluta o relativa, y en particular, conocer el papel que la sílaba juega en la codificación de la información posicional dentro de las representaciones ortográficas. Para ello, empleamos dos experimentos con una tarea de solución de anagramas. En dicha tarea, se presenta un anagrama (CSIAGTO) y el participante tiene que descubrir la palabra que se forma ordenando las letras internas del anagrama (CASTIGO). Los resultados del Experimento 1 muestran un efecto de la presencia de bigramas adyacentes: los anagramas que mantienen un bigrama en una posición adyacente (CTGASIO) son resueltos con más frecuencia que aquellos con todas las letras internas desordenadas (CSIAGTO), lo que sugiere que para reconstruir una secuencia altamente distorsionada, es importante conocer la posición relativa de una letra con respecto a otra. En el Experimento 2, manipulamos la naturaleza silábica o subsilábica del bigrama para estudiar si el efecto de bigrama puede reducirse a un efecto silábico o puede estar modulado por el estatus subléxico (silábico o subsilábico) del bigrama. Los resultados obtenidos muestran un efecto silábico. Finalmente, examinamos las implicaciones de estos resultados para los modelos actuales de reconocimiento visual de palabras.
\end{abstract}

Palabras Clave: Información posicional; unidades subléxicas; tarea de solución de anagramas.

\section{Introducción}

En los últimos años ha habido un creciente interés en el estudio de la codificación de la posición de cada una de las letras que forman una palabra. Dicho interés ha surgido en buena medida con la aparición de un fenómeno empírico como es el efecto de transposición de letras (secuencias en las que se transponen dos letras): ítems creados por transposición de dos letras como meczla se confunden fácilmente con su palabra-base (mezcla). Dicho fenómeno cuestiona el esquema de codificación de la estructura posicional de las representaciones ortográficas propuesto por los modelos tradicionales de reconocimiento visual de palabras (véase McClelland \& Rumelhart, 1981; Coltheart, Rastle, Perry, Langdon \& Ziegler, 2001; Grainger \& Jacobs, 1996). En estos modelos, cada unidad de codificación se encarga de recoger información de la identidad de la letra en una posición específica (codificación estricta de la posición, ver Figura 1, Gráfico a). Sin embargo, un elevado número de experimentos, empleando diferentes metodologías e idiomas, han mostrado que la presentación de pseudopalabras creadas por transposición de dos letras adyacentes (o no adyacentes; v.g., privamera - primavera) activan la representación léxica de su palabra base (v.g., Perea \& Lupker, 2003a, 2003b, 2004; véase también Acha \& Perea, 2008, 2010; García-Orza, Perea \& Muñoz, 2011; Lupker, Perea, \& Davis, 2008; Perea \& Acha,

* Dirección para correspondencia [Correspondence address]: Ascensión Pagán. School of Psychology. Shackleton Building (B44). University of Southampton. Highfield Campus. Southampton SO17 1BJ (United Kingdom). E-mail: a.p.pagan-camacho@soton.ac.uk
Title: The role of the syllable in positional information coding in orthographic representations.

Abstract: The main aims of this study are: i) to examine whether the positional information is coded in absolute or relative terms in orthographic representations, and ii) to explore the role of syllabic units in this process. To that end, we will use a new technique: the Anagram Solution Task (AST). In an AST, an anagram is presented (e.g., CSIAGTO) and the participant has to mentally rearrange the inner letters of the word (CASTIGO -punishment, penalty). In Experiment 1, the results showed a bigram effect: the anagrams that maintained a bigram in an adjacent position (CTGASIO) were solved with higher accuracy than those anagrams that had all inner letters disordered (CSIAGTO). That is, relative position is more effective than absolute position to reconstruct a highly disordered anagram. In Experiment 2, we examined whether or not this bigram effect had a syllable nature by manipulating syllabic bigram structure. Results showed a syllabic effect. Taken together, the data have implications for the current models of visual-word recognition.

Key words: Letter position; sublexical units; anagram solution task.

2010; Perea \& Carreiras, 2006a, 2006b, 2006c, 2008; Perea, Abu Mallouh \& Carreiras, 2010; Perea, Duñabeitia \& Carreiras, 2008; Perea \& Estévez, 2008; Perea \& Pérez, 2009; Perea, Winskel \& Ratitamkul, 2012; Schoonbaert \& Grainger, 2004; Velan \& Frost, 2011). Es importante subrayar que estos efectos no se restringen a "tareas de laboratorio", sino que también se han obtenido en tareas de lectura de textos en las que se recogen los movimientos oculares (véase Acha \& Perea, 2008; Johnson, Perea \& Rayner, 2007; Johnson, 2009; Perea, Nakatani \& van Leeuwen, 2011; Rayner, White, Johnson \& Liversedge, 2006).

Debido a la robustez de los efectos de transposición de letras, han sido propuestos un buen número de modelos de reconocimiento visual de palabras que permiten una codificación más flexible de las letras en una palabra, y que pueden acomodar dicho efecto. De manera esquemática, dichos modelos puede clasificarse en tres familias. En una familia de modelos, la opción ha sido asumir que existe cierta incertidumbre perceptiva respecto a la posición de las letras dentro de las palabras, como en el caso del modelo de solapamiento ("Overlap model", Gómez, Perea \& Ratcliff, 2008), y que ha sido un supuesto adoptado en el modelo de "Bayesian Reader" (Norris, Kinoshita \& van Casteren, 2010). El modelo de solapamiento asume que en una secuencia que se presenta sin máscara y con un tiempo de exposición ilimitado, se produce una codificación precisa de la información posicional. Sin embargo, cuando la secuencia se presenta brevemente, la codificación de la posición de cada letra está sometida a cierto grado de incertidumbre lo que se representa en el modelo mediante una distribución de la identidad de las letras sobre las posiciones en la secuencia (supuesto de incerti- 
dumbre de la posición). En este caso, la información sobre los rasgos visuales de las letras está distribuida espacialmente (ver Figura 1, gráfico c). Por ejemplo, si tenemos la palabra "ALMA", la letra "M" se asocia en mayor grado a la tercera posición pero también a las otras posiciones, primera, segunda y cuarta. Lo mismo ocurriría con las letras "A" y " $L$ ". La similitud ortográfica en este modelo se calcula comparando dos secuencias de letras y calculando la cantidad de solapamiento que existe entre las dos.

En una segunda familia de modelos se asume un esquema de codificación contextual como es el caso del modelo de bigrama abierto ("Open Bigram") de Grainger \& van Heuven (2003; véase también Grainger, Granier, Fariolli, van Assche \& van Heuven, 2006) y el modelo SERIOL de Whitney (2001) (ver Figura 1, gráfico d). Estos modelos codifican la información de la posición relativa de las letras a través de parejas de letras (bigramas), y establecen el grado de similitud ortográfica a través del solapamiento que existe entre los bigramas de las dos palabras que se comparan: cuantos más bigramas compartan ambas secuencias, mayor similitud ortográfica habrá. Siguiendo el mismo ejemplo, se activaran todos los pares de letras que se pueden formar con la palabra "ALMA": "AA", "AL", "AA", "LM", "AM", "MA", "LA" y "A_"; además, aquellos bigramas formados por letras en posiciones adyacentes se activarán en mayor medida que los que estén formados por letras no adyacentes (“_A", "AL", "LM", "MA" y "A_" serán los que reciban mayor activación), y finalmente, en el modelo SERIOL, además de lo anterior, se emplea también, un esquema de codificación espacial donde el bigrama que aparece en primer lugar es el que tiene mayor activación, el segundo bigrama es el segundo más activado, etc.

Finalmente, una tercera opción es el modelo SOLAR (Davis, 1999; 2010), en el que también se emplea el esquema de codificación espacial pero sin tener en cuenta la información contextual como en el modelo SERIOL, de manera que la letra que va en primer lugar es la que tiene mayor activación, la segunda letra es la segunda que tiene más activación, etc. (ver Figura 1, gráfico b). El modelo SOLAR define el grado de similitud ortográfica en función de los niveles de activación de las letras que forman la secuencia, de tal manera que dos palabras que tengan las mismas letras, tendrán las mismas letras activadas pero los patrones de activación serán diferentes.

El objetivo de este trabajo consiste en aportar evidencia empírica sobre el fenómeno de la codificación de la posición de las letras. En particular, queremos estudiar, en primer lugar, si la codificación de la posición de las letras se realiza de forma absoluta o relativa, es decir, si se tiene o no en cuenta la información de la posición de unas letras con respecto a otras; y en segundo lugar, si esta información es más discriminativa por su naturaleza subléxica, en particular, sobre el papel de la sílaba. Hay modelos de reconocimiento de palabras que proponen un nivel de representación intermedio en el que se codifica la información subléxica (silábica y morfológica) que participa en el acceso al léxico (Ans, Carbonnel
\& Valdois, 1998; Ferrand, Seguí \& Grainger, 1994; Ferrand, Grainger \& Seguí 1996; Conrad, Tamm, Carreiras \& Jacobs, 2010). En español, existen numerosos estudios que muestran que la sílaba puede actuar como una unidad de naturaleza fonológica y ortográfica durante el acceso al léxico (v.g., Álvarez, Carreiras \& Perea, 2004; Carreiras, Álvarez \& de Vega, 1993; Perea \& Carreiras, 1998, por citar tres estudios sobre este tema). Es importante señalar que, por un lado, ninguno de los modelos de reconocimiento visual de palabras (v.g., modelos de solapamiento, bigrama abierto o de codificación espacial), que puede acomodar efectos de transposición de letras, emplea niveles de representación subléxico de tipo silábico o morfémico; y por otro lado, que los modelos que representan un nivel silábico no pueden acomodar los efectos de transposición de letras.
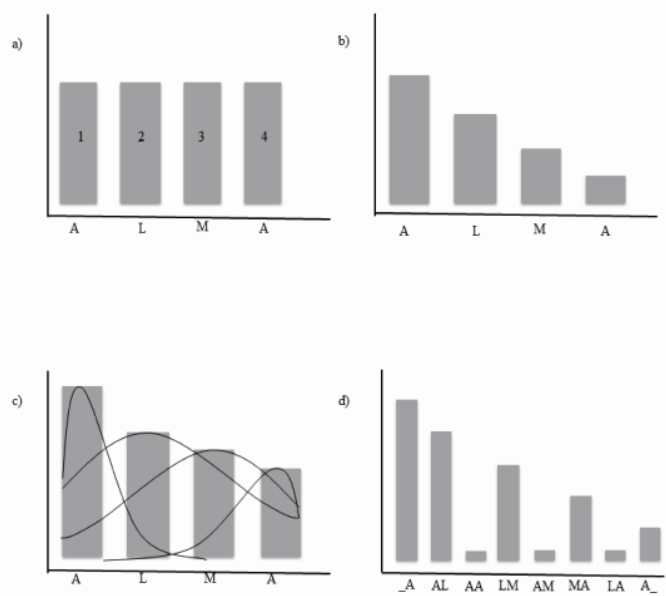

Figura 1. Codificación de la información posicional según los distintos modelos y esquemas de codificación: a) Esquema de codificación estricta (v.g. McClelland \& Rumelhart, 1981); b) Esquema de codificación espacial (modelo SOLAR, Davis, 1999, 2010); c) Modelo de solapamiento (Gómez, et al., 2008); y d) Esquema de codificación contextual (v.g., modelo SERIOL; Whitney, 2001).

Hasta ahora, la mayor parte de los resultados empíricos descritos en estudios sobre la información posicional procede de tareas en las que las respuestas del sujeto se realizan a estímulos que son muy similares (v.g., transposición de letras adyacentes: caisno - CASINO o de letras no adyacentes: ca-

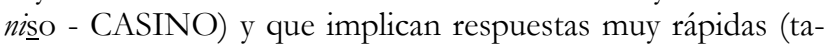
rea de decisión léxica, de lectura en voz alta -naming, y recientemente, la tarea de igual-diferente García-Orza, Perea \& Muñoz, 2011; Muñoz, Perea, García-Orza \& Barber, 2012) dentro de un paradigma de priming enmascarado (donde se presenta una máscara visual (\#\#\#\#) durante 500 ms., un secuencia de letras (prime) durante $60 \mathrm{~ms}$., y por último, la palabra (target) a la que se debe responder). Estas tareas permiten estudiar con gran precisión los detalles de la codificación de la información posicional en las representaciones ortográficas. En estos casos, la alteración de la posición de dos letras de la secuencia da lugar a efectos de priming que son muy pequeños en magnitud. Sin embargo, si realizáramos cambios en la posición de un mayor numero de letras de la 
secuencia (dando como resultado un material muy poco similar con el estimulo base) y utilizando estas mismas tareas con priming enmascarado, sería muy difícil encontrar efectos de priming.

Una excepción es el trabajo Guerrera \& Foster (2008), en el que se manipularon múltiples transposiciones de letras adyacentes en palabras de 8 letras para comprobar el grado de distorsión (disimilitud) que es tolerado por el sistema de reconocimiento de palabras, y emplearon una tarea de decisión léxica estándar con priming enmascarado. Guerrera \& Forster encontraron un efecto de priming mayor en los primes que solo mantenían dos letras en su posición original (13254768, 12436587; v.g., la palabra "sidewalk" es representada como 12345678; a la secuencia de letras "sdiwelak, creada por la transposición de todas las letras excepto la primera y la ultima de esa palabra, le corresponde 13254768; los números describen el lugar que ocupaba cada una de las letras del prime en la palabra base) frente a los primes que tenían todas las letras desordenadas $(21436587,43218765)$. El efecto de priming era mayor para aquellos primes que conservaban las letras externas en su posición (132544768 y 12436587). Guerrera \& Forster concluyeron que el sistema de reconocimiento visual de palabras es particularmente flexible en la codificación de la información posicional y sugieren la idea de que las letras externas juegan un papel más influyente en el reconocimiento de las palabras (véase Perea \& Lupker, 2003a; 2003b; 2007, para más evidencia sobre el papel de las letras externas).

En este estudio, hemos utilizado una tarea de solución de anagramas (v.g., se pide al participante que indique la palabra que subyace en la secuencia CSLAGTO). La razón es que esta tarea permite mayor flexibilidad a la hora de manipular las posiciones de varias letras a la vez, de modo que podemos obtener evidencia convergente sobre cómo la información posicional se codifica dentro del léxico ortográfico. La tarea de solución de anagramas se ha utilizado para evidenciar una gran variedad de fenómenos relacionados tanto con el reconocimiento de palabras como con los mecanismos de solución de problemas (Mayzner \& Tresselt, 1962; Schwartz, 1968; Topper, Macey \& Solso, 1973; Tresselt \& Mayzner, 1968; Velandrino, 1985). Esta técnica consiste en la presentación de una secuencia de letras que reordenadas de una determinada manera forman una palabra. La tarea del participante es descubrir la palabra que se encuentra escondida en el anagrama. Esta técnica permite realizar manipulaciones experimentales que no podrían ser puestas a prueba con los paradigmas más comunes como la técnica de priming enmascarado -dado que como hemos señalado arriba, los efectos con priming enmascarado son típicamente muy pequeños y se utiliza en manipulaciones sutiles (véase Gómez, et al., 2008, para discusión de este tema). Para comprender mejor qué es lo que ocurre cuando el sujeto tiene que resolver un anagrama, proponemos un posible esquema de procesamiento que está basado en el Modelo clásico de Activación de Becker $(1976,1979,1985)$ y que fue desarrollado por Paap, Newsome, McDonald \& Schvaneveldt
(1982). Cuando se presenta un anagrama, se activan una serie de representaciones ortográficas léxicas con las que se elabora una lista de candidatos. La activación de estos candidatos se realiza en función de diversos criterios, como por ejemplo, el número de letras que comparten, la frecuencia de las letras y/o las secuencias intermedias (bigramas, sílabas, morfemas), la similitud ortográfica, etc. De este modo se selecciona una representación ortográfica y se produce una comparación post-acceso entre el candidato y el input presentado. Si no coincide la representación ortográfica con el input, entonces comienza otra comparación post-acceso con otro candidato de la lista pre-activada. Sin embargo, si ambos coinciden, entonces el sujeto tiene la solución del anagrama. La solución del anagrama será correcta en algunos casos pero no en otros. Probablemente, los errores se producen por una gran similitud entre las diferentes representaciones ortográficas activadas por el anagrama.

\section{Experimento 1}

El primer objetivo de este trabajo es estudiar qué tipo de información posicional (individual o relativa) es eficaz para identificar una palabra en una tarea de solución de anagramas. Por ejemplo, para la palabra CASTIGO, podemos formar dos anagramas con el mismo grado de similitud con la palabra solución (CSIAGTO y CTG ASIO). La diferencia entre el primer y el segundo anagrama es que en el primero, todas las letras internas se han desplazado de su posición original, mientras que en el segundo, además de ese desplazamiento se ha conservado la posición relativa de dos letras de la secuencia (bigrama). Si la información relativa está implicada en la codificación de la información posicional, deberíamos observar una ventaja para la solución de aquellos anagramas que contengan esta información. Es importante recordar que en modelos como el SOLAR y el de solapamiento, la información posicional se codifica según la posición individual de cada uno de los caracteres. Sin embargo, en ninguno de estos modelos se incluye de modo explícito información posicional que relacione cada letra con las letras adyacentes, información que sí incluyen los modelos SERIOL y Bigrama Abierto. En el Experimento 1, estudiaremos si los anagramas que conservan un bigrama de la secuencia original (codificación relativa de la posición) se resuelven mejor (mayor número de soluciones correctas) que aquellos que no conservan ninguno de los bigramas de la secuencia original (codificación individual de la posición). Además se manipulará la frecuencia de uso de las palabrasbase, para observar los posibles efectos de la activación léxica en la solución de anagramas.

\section{Método}

Participantes. En este experimento participaron 48 estudiantes de la asignatura de Psicología del Lenguaje de $4^{\circ}$ curso de la Licenciatura de Psicología de la Universidad de Murcia a cambio de un crédito en clases prácticas. 
Diseño y material. El material estaba formado por 48 anagramas de 7 letras de longitud. Los anagramas mantenían en su posición original la primera y la última letra, mientras que todas las letras internas estaban desordenadas (v.g., JOADGUR - JUGADOR). Todos los anagramas tenían una solución única. Las variables manipuladas ortogonalmente fueron tres. En primer lugar, la Frecuencia Léxica: la mitad de palabras eran de frecuencia alta (media $=78.66$ tokens por millón) y la mitad de baja frecuencia (media $=0.39$ tokens por millón) según el corpus LEXESP (Sebastián, Martí, Cuetos, \& Carreiras, 2000) de la base B-Pal (Davis \& Perea, 2005). El segundo factor manipulado fue la presencia o ausencia de información relacional en el anagrama. En concreto, cada palabra generaba dos anagramas alternativos. En uno de ellos, el anagrama conservaba un bigrama de la palabra original pero en una posición adyacente a la posición original que tenía el bigrama en la palabra base (como en CTG ASIO para la palabra CASTIGO; fijese que la única información que se conserva es la posición relativa: la letra A precede a la letra S, pero no la posición absoluta: las letras AS aparecen en segunda y tercera posición en la palabra original mientras que en el anagrama aparecen en cuarta y quinta), en el otro, el anagrama no conservaba ningún bigrama (como en CSIAGTO). Se controló que el grado de similitud entre los anagramas y la palabra original no variara para los dos grupos de palabras (con y sin bigrama). El tercer factor fue el grado de distorsión de la secuencia de letras, entendido como el grado de similitud entre el anagrama y la palabra original. El grado de similitud hace referencia a la diferencia entre la posición que ocupan las letras en el anagrama con respecto a la posición que esas mismas letras tienen en la palabra base. Para calcular el grado de similitud/distorsión de cada anagrama se utilizó el programa Match Calculator incluido en el modelo SOLAR que arroja los valores de similitud definidos por los modelos SOLAR, SERIOL y Bigrama Abierto. Teníamos dos niveles, alta distorsión (baja similitud con la palabra original, promedio $=.59$ ) o baja distorsión (alta similitud con la palabra original, promedio $=.45$ ). Se eligieron diferentes secuencias desordenadas para cada uno de los ítems de cada condición, pero el grado de desorden era similar calculado según el programa Match Calculator. No había diferencias estadísticamente significativas entre las diferentes distorsiones dentro de la condición (alta o baja distorsión). Un ejemplo es: CASTIGO - CSIAGTO (baja distorsión $=.36$, ausencia de bigrama) - CTGASIO (baja distorsión $=.44$, presencia del bigrama); MALETIN - MTELIAN (alta distorsión $=.64$, ausencia del bigrama) - MIETLAN (alta distorsión $=.58$, presencia del bigrama).

Procedimiento. Los participantes realizaron la tarea de forma individual en una habitación silenciosa y correctamente iluminada. Los anagramas se presentaron en letras mayúsculas en el centro de la pantalla de un ordenador y durante un tiempo máximo de 30 segundos. El participante tenía que encontrar la palabra objetivo (fuente del anagrama) antes de que finalizara la exposición del anagrama. En caso de encontrar la solución debía pulsar la barra espaciadora para que el anagrama desapareciera de la pantalla y entonces, escribir en el ordenador la palabra solución del anagrama presentado. Al finalizar cada ensayo se suministró información sobre la solución del anagrama y comenzaba el siguiente ensayo. Para evitar los efectos debidos a la repetición de un ítem (anagrama con y sin bigrama), se crearon dos listas contrabalanceadas donde los anagramas fueron presentados aleatoriamente a cada uno de los participantes.

\section{Resultados y Discusión}

Se realizó un análisis de varianza (ANOVA) según un diseño 2 (frecuencia léxica: alta vs. baja) x 2 (presencia/ausencia de bigramas de la palabra-base) x 2 (grado de distorsión: alto vs. bajo) sobre el porcentaje de aciertos en la tarea. Tanto la frecuencia léxica como la distorsión se introdujeron en el análisis como factores intrasujetos mientras la presencia o ausencia del bigrama fue analizada como factor intersujetos en el análisis por sujetos. En el análisis por ítems los factores frecuencia y distorsión se manipulan intersujetos y la presencia/ausencia de bigrama como factor intrasujetos. Las medias y errores típicos de los porcentajes de aciertos de los sujetos se presentan en la Tabla 1.

Tabla 1. Medias y (errores típicos) de los porcentajes de aciertos en la tarea de solución de anagramas en el Experimento 1.

\begin{tabular}{llll}
\multirow{3}{*}{ FL Alta } & Dist Alta & Sin Bigrama & Con Bigrama \\
\cline { 3 - 4 } FL Baja & Dist Baja & $53.74(3.38)$ & $58.16(3.64)$ \\
& Dist Alta & $44.90(3.30)$ & $69.05(3.70)$ \\
& Dist Baja & $40.48(3.47)$ & $48.30(3.16)$ \\
\hline
\end{tabular}

El ANOVA mostró, en primer lugar, que los sujetos resolvían mayor número de anagramas procedentes de palabras de alta frecuencia que de palabras de baja frecuencia $(d$ $=14 \%), F 1(1,48)=44.36, p<.01, \eta^{2} p=.48 ; F 2(1,44)=4.46$, $p=.04, \eta_{p}^{2}=.09$. En segundo lugar, los anagramas que conservaban un bigrama de la palabra solución se resolvieron mejor que los que no lo mantenían $(d=8 \%), F 1(1,48)=$ $10.54, p<.00, \eta^{2}{ }_{p}=.18 ; \mathrm{F} 2(1,44)=3.80, p<.06, \eta^{2}{ }_{p}=.08 . \mathrm{El}$ factor principal grado de distorsión no mostró un efecto significativo (ambas Fs $<1$ ). Dos de las interacciones de este factor resultaron significativas: distorsión por bigrama $\left(F 1(1,48)=4.03, p=.05, \eta_{p}^{2}=.11 ; F 2(1,44)=0.53, p=.47\right.$, $\left.\eta_{p}^{2}=.01\right)$ y frecuencia léxica por distorsión $(F 1(1,48)=5.91$, $\left.p<.02, \eta_{p}^{2}=.08 ; F 2(1,44)=0.331, p=.57, \eta^{2}=.01\right)$. En el caso de la interacción distorsión x bigrama, el bigrama facilitó en mayor grado la respuesta del sujeto en los anagramas poco distorsionados $(F 1(1,48)=10.19, p<.01)$ que cuando se trataba de anagramas con una alta distorsión $(F 1(1,48)=$ $2.88, p=.1)$. La interacción de frecuencia $\mathrm{x}$ distorsión se debe a que la similitud entre el anagrama y la palabra solución influye en la solución de anagramas procedentes de palabras de alta frecuencia léxica $(F 1(1,48)=5.79, p=.02)$ pero no en las procedentes de palabras de baja frecuencia $(F 1(1,48)$ $=1.04, p=.31)$. Los anagramas de palabras de alta frecuencia producen un acceso léxico más rápido que las de baja 
frecuencia y si además, los anagramas están poco distorsionados (son muy similares a la palabra original) significa que la competición entre candidatos es menor y es más fácil encontrar la solución. Por último, ni la interacción de frecuencia $x$ bigrama $\left(F 1(1,48)=1.022, p=.32, \eta_{p}^{2}=.02 ; F 2(1,44)=\right.$ $0.331, p=.57, \eta^{2}{ }^{2}=.01$ ) ni la triple interacción (frecuencia $\mathrm{x}$ distorsión x bigrama $\left(F 1(1,48)=0.468, p=.50, \eta^{2}=.01\right.$; $\left.F 2(1,44)=1.062, p=.31, \eta^{2}=.02\right)$ resultaron significativas.

En resumen, en este experimento hemos encontrado un efecto de la información relacional en la tarea de solución de anagramas que viene modulado por el estatus léxico de la palabra solución. Este resultado sugiere que la información posicional relativa es más eficaz a la hora de identificar una palabra que la información individual de cada carácter, tal y como predicen los modelos SERIOL y Bigrama Abierto. Obsérvese que, si bien dicho resultado aparentemente apoya más los modelos SERIOL y Bigrama Abierto que los modelos SOLAR o de solapamiento, la idea de una información de corte posicional está implícita en estos dos últimos modelos. Otro resultado de interés es que hemos mostrado que tanto el efecto de la información relacional como el efecto de la frecuencia léxica aparecen cuando la distorsión del anagrama es baja (muy similar a la palabra original) pero no cuando es alta (disimilar a la palabra original). Esto podría indicar que los participantes pueden estar utilizando dos estrategias diferentes en la tarea de solución de anagramas. En el caso de los anagramas poco distorsionados, los participantes podrían utilizar una estrategia que se apoya en la activación léxica consistente en chequear como posibles soluciones aquellas representaciones que están más activas en su léxico. Sin embargo, con los anagramas muy distorsionados, al no alcanzar el nivel mínimo de activación de entradas léxicas, no habría propuestas de solución para el anagrama y en este caso, se impondría una estrategia más deductiva, basada posiblemente en la elaboración de soluciones a partir de la combinatoria de las letras. Por último, la ausencia de significación en la interacción triple (Distorsión x Bigrama x Frecuencia) podría indicar que los efectos obtenidos suceden en diferentes momentos del procesamiento si seguimos la lógica de factores aditivos de Sternberg (1969). El efecto de la información relacional afectaría a un estadio inicial donde se codifica una representación de acceso, mientras que el efecto de frecuencia léxica afectaría a un estadio posterior donde se produce el acceso léxico.

\section{Experimento 2}

En el experimento anterior, hemos encontrado un efecto de la información posicional relativa, en el que observamos una mayor tasa de aciertos en anagramas que conservan un bigrama del estímulo original que en aquellos casos en los que no lo mantienen. En este segundo experimento, estudiamos si los efectos obtenidos en el Experimento 1 pueden explicarse a partir del estatus silábico de algunos de los bigramas. Existen numerosos estudios que proponen la sílaba como unidad intermedia que facilita el acceso al léxico (Prinzmetal,
Treiman \& Rho, 1986; Carreiras, et al., 1993; Ferrand, et al., 1994; 1996; Hutzler, Bergmann, Conrad, Kronbichler, Stenneken \& Jacobs, 2004; Perea \& Carreiras, 1998; Quintanilla, 2006) frente a otras unidades intermedias (Álvarez, Carreiras \& Taft, 2001; Álvarez, Carreiras \& Perea, 2004). Perea \& Carreiras (2006a) utilizaron una tarea de decisión léxica para saber si pseudopalabras creadas por dos sílabas transpuestas (privemara - PRIMAVERA) son más difíciles de rechazar que pseudopalabras formadas por dos bigramas transpuestos (primerava - PRIMAVERA). Los datos mostraron un efecto de similitud cuando se transponían dos sílabas CV internas pero dicho efecto no difería del efecto de similitud encontrado con bigramas CV no silábicos. Perea \& Carreiras interpretaron este efecto como una prueba de que la posición de letras internas se codifica de forma flexible, pero al no encontrar diferencias en el efecto de similitud cuando transponían un bigrama silábico o un bigrama no silábico, propusieron que los efectos de transposición ocurrían en un nivel anterior: en el nivel grafémico (letra). Estos resultados parecen contradecir la hipótesis de que el estatus silábico de los bigramas intervenga en la codificación posicional de las representaciones silábicas. Sin embargo, existen varios motivos que justifican realizar un nuevo experimento para probar esta hipótesis. Como anteriormente hemos referido, resulta sorprendente que una estructura subléxica que ha recibido un apoyo empírico tan consistente como unidad intermedia en el acceso a las representaciones léxicas en español se encuentre al margen de la codificación de las representaciones que sirven para tal propósito. En segundo lugar, el hecho de que los autores no encuentren un efecto de la sílaba no significa que no tenga un papel relevante en la codificación de la información posicional, sino que quizás la tarea empleada no ha sido sensible para detectar un efecto silábico en pseudopalabras; recordemos que el efecto de la sílaba podría ser menos importante para unidades sin representación léxica que para las palabras (Carreiras, et al., 1993). Finalmente, existen importantes diferencias entre la tarea de decisión léxica y la tarea de solución de anagramas, por lo que cabe considerar la posibilidad de que las unidades silábicas puedan mostrar su efecto en una tarea que (1) implica mayor tiempo de procesamiento de input antes de producir una respuesta y (2) enfoca la atención del sujeto sobre el estatus léxico del input y no en su aceptación o rechazo.

En definitiva, el objetivo de este experimento es estudiar si el estatus silábico de los bigramas facilita la solución de anagramas en mayor medida que cuando el bigrama carece de dicho estatus. Por tanto, si la sílaba funciona como una unidad de acceso léxico en español, aquellos anagramas que mantienen un bigrama silábico serán identificados en mayor proporción que aquellos anagramas que conservan un bigrama no silábico. Dicho resultado implicaría que los modelos de reconocimiento de palabras como el SERIOL, de bigrama abierto, SOLAR, o solapamiento tendrían que ser modificados para poder acomodar los efectos de corte silábico -recordemos que estos modelos asumen únicamente un nivel de letras (o de bigramas) pero no un nivel de sílabas, 
por lo que dichos modelos no predicen efecto alguno de las representaciones silábicas en la solución de anagramas.

\section{Método}

Participantes. En este experimento participaron 86 estudiantes de $4^{\circ}$ curso de la Licenciatura de Psicología de la Universidad de Murcia a cambio de un crédito de prácticas.

Diseño y Materiales. Los materiales estaban formados por 30 anagramas de solución única de 7 letras de longitud. Los anagramas se construyeron variando la posición de todas las letras interiores de la palabra original, conservando en su posición la primera y última letra. Se manipuló la frecuencia léxica de las palabras fuente: la mitad fueron de alta frecuencia (media $=117.13$ tokens por millón) y la otra mitad de media frecuencia (media $=46.93$ tokens por millón) en el corpus LEXESP (Sebastián, et al., 2000) de la base B-Pal (Davis \& Perea, 2005). El segundo factor manipulado fue el status del bigrama (silábico o subsilábico). La selección de cada palabra se produjo de tal forma que existiera otra palabra que en idéntica posición presentara la misma secuencia de caracteres (bigrama) pero difiriera en el estatus silábico de dicha secuencia. Por ejemplo, en la pareja ASUMIDO USUARIO, tenemos que el bigrama [SU] aparece en ambas palabras, pero mientras que en el primer elemento del par (ASUMIDO) es una sílaba de la palabra, en el segundo (USUARIO) la secuencia no constituye una sílaba. El bigrama que se manipulaba era el que correspondía con la segunda sílaba de la palabra porque manteníamos la primera y la última letra en su posición original, lo que anulaba la posibilidad de mantener el primer bigrama de la palabra. Cabe señalar aquí que algunos estudios en español han mostrado que no solo la primera sílaba sino también la segunda sílaba en palabras bisílabas y trisílabas muestran efectos facilitatorios (Álvarez, et al., 1998; 2000; Hutzler, et al., 2004)). Todos los bigramas tenían una estructura CV (consonante-vocal). Cada pareja de palabras tenía el mismo parecido a las palabras originales, es decir, el desorden interno de la letras seguía el mismo patrón en las dos palabras de la pareja, según el modelo SOLAR (Davis, 1999).

Procedimiento. Se siguió un procedimiento similar al empleado en el experimento anterior. Se creó una única lista que fue presentada aleatoriamente a cada uno de los participantes.

\section{Resultados y discusión}

Se realizó un análisis de varianza (ANOVA) según un diseño 2 (frecuencia léxica: alta vs. media) x 2 (estatus del bigrama: silábico vs. subsilábico) sobre el porcentaje de aciertos en la tarea. En el análisis por sujetos, la frecuencia léxica se analizó como factor intrasujetos mientras que la variable estatus silábico o subsilábico del bigrama fue analizada como factor intersujetos. En el análisis por ítems, la frecuencia es analizada como factor intersujetos y el estatus silábico como factor intrasujetos. En la Tabla 2 se muestran las medias y los errores típicos de los sujetos en este experimento para las diferentes combinaciones factoriales del diseño.

Tabla 2. Medias y (errores típicos) de los porcentajes de aciertos en la tarea de solución de anagramas en el Experimento 2.

\begin{tabular}{lll}
\hline & Alta frecuencia & Media frecuencia \\
\cline { 2 - 3 } Bigr Silábico & $63.81(1.84)$ & $60.13(2.03)$ \\
Bigr Subsilábico & $50.58(2.24)$ & $43.19(2.19)$ \\
\hline
\end{tabular}

El ANOVA mostró que las palabras de alta frecuencia se resuelven mejor que las de media frecuencia $(d=5.54 \%)$, diferencia que fue significativa en el análisis por sujetos, $F 1(1,85)=8.64, p=.00, \eta^{2}=.45$, pero no en el análisis por ítem $\left(F 2(1,13)=0.31, p=.59, \eta_{p}^{2}=.02\right)$-recuérdese que la manipulación de frecuencia de uso fue relativamente débil (el promedio de las palabras de media frecuencia fue más de 40 por millón). Pero el resultado de mayor importancia en este experimento es que aquellos anagramas cuyos bigramas representan una sílaba de la palabra fuente fueron resueltos mejor $(d=15.1 \%)$ que aquellos en los que el bigrama no representa una sílaba de la palabra fuente $(F 1(1,85)=68.81, p$ $\left.=.00, \eta_{p}^{2}=.09 ; F 2(1,13)=7.34, p<.02, \eta_{p}^{2}=.36\right)$. La interacción entre ambos factores no alcanzó los niveles de significación $\left(F 1(1,85)=1.382, p=.24, \eta_{p}^{2}=.02 ; F 2(1,13)=\right.$ $\left.0.111, p=.74, \eta_{p}^{2}=.01\right)$.

En resumen, en este experimento, hemos encontrado que cuando en un anagrama mantenemos un bigrama con estatus silábico (segunda sílaba de la palabra) en una posición adyacente, los anagramas se resuelven mejor que cuando es un bigrama subsilábico. Dicho resultado es consistente con los efectos silábicos encontrados en otros estudios que consideran la sílaba como unidad intermedia que facilita el acceso al léxico (Carreiras, et al., 1993; Carreiras \& Perea, 2002; Hutzler, et al., 2004; Álvarez, de Vega \& Carreiras, 1998; Álvarez, Carreiras \& de Vega, 2000; Perea \& Carreiras, 1998).

\section{Discusión general}

El objetivo principal de esta serie de experimentos es mostrar evidencia empírica de cómo se codifica la información posicional en el léxico ortográfico. La tarea de solución de anagramas nos ha permitido manipular la posición de varias letras a la vez en cadenas altamente distorsionadas.

En el Experimento 1, hemos encontrado que aquellos anagramas que mantienen un bigrama en una posición adyacente se resuelven mejor que aquellos que mantienen todas sus letras internas desordenadas. En primer lugar, este efecto proporciona evidencia convergente con la idea de que la codificación de las letras internas de una palabra se realiza de forma flexible y distribuida, ajustándose a las predicciones que realizan los modelos basados en un esquema de codificación flexible de la posición de las letras (modelos de solapamiento, SERIOL, bigrama abierto, y SOLAR). En segundo lugar, nuestros datos señalan la importancia de la información sobre la posición relativa entre las letras en el reconocimiento visual de palabras, tal y como predicen los mo- 
delos SERIOL y Bigrama Abierto. Además, según el modelo de la tarea que proponíamos en la introducción, observamos que la conservación del bigrama en una posición adyacente a la original ayuda a seleccionar al candidato adecuado en el proceso de comprobación post-acceso, lo que indica una prueba a favor de aquellos modelos que contienen un nivel de representación de tipo subléxico (v.g., Ans, et al. 1998; Conrad, et al. 2010; Ferrand, et al., 1994; 1996) que facilita la búsqueda de las representaciones ortográficas léxicas. El hecho de que los efectos encontrados en el primer experimento se puedan explicar según los diferentes modelos de reconocimiento visual de palabras valida la tarea de solución de anagramas como una técnica apropiada para el estudio de la codificación de la posición de las letras.

En el Experimento 2, los datos señalan que el estatus silábico de los bigramas influye en la identificación de palabras, en concreto, hay una mayor facilitación de los bigramas de tipo silábico. Este resultado contiene las mayores implicaciones teóricas del presente trabajo porque ninguno de los modelos que se basan en un esquema flexible de codificación (como los modelos que emplean bigramas como unidades mediadoras entre las letras y las palabras) es capaz de dar cuenta de dicho efecto. La razón es simple: tanto el modelo de Bigrama Abierto como SERIOL codifican de forma explícita los bigramas como unidades que recogen la posición relativa de la secuencia pero no distinguen en sus predicciones entre un bigrama (dos letras adyacentes) y un bigrama silábico, ya que no incluyen un nivel de representación subléxica de tipo silábica (ni tampoco morfémica). Recordemos que estos modelos asumen que la única información importante para el procesamiento ortográfico es la letra o el bigrama. Sin embargo, estos modelos no tienen en cuenta que estas letras o bigramas pueden tener una función diferente dentro de la secuencia. Esto supone un problema para estos modelos -al menos en lenguas como el español- ya que ignoran el valor lingüístico de las unidades de codificación silábica que son utilizadas en lenguas con transparencia ortográfica y con límites silábicos claros (véase Lee \& Taft, 2009, 2011, para evidencia de efectos de transposición de corte subsilábico en coreano).

Por tanto, los resultados del segundo experimento implican que los modelos de reconocimiento de palabras que tienen un esquema flexible de codificación de letras deben incorporar un nivel subléxico de corte silábico para poder dar cuenta de nuestros resultados. ¿Implica ello que los esquemas de codificación ortográficos del modelo SERIOL, del modelo de bigrama abierto, de solapamiento o del modelo SOLAR son erróneos y deben sustituirse por un esquema que tenga en cuenta el nivel silábico de procesamiento? La respuesta provisional sería "no". Es muy probable que los estadios más iniciales de procesamiento vengan ligados a un procesamiento de corte ortográfico, mientras que ligeramente después aparecerían los efectos de corte fonológico. Es en este segundo momento donde unidades fonológicas, como es la sílaba, jugarían un papel relevante (Álvarez, et al., 2004). Por tanto, lo que los modelos de reconocimiento de palabras como el solapamiento, SERIOL, bigrama abierto y SOLAR necesitan es una especificación de los procesos fonológicos durante el reconocimiento visual de palabra. El modelo SERIOL ofrece una primera aproximación al procesamiento fonológico (véase Whitney \& Cornelissen, 2005), pero que no incluye las sílabas como unidades, mientras que los otros modelos únicamente incluyen el módulo ortográfico. Creemos que las futuras implementaciones de estos modelos necesitan incluir un nivel fonológico, no solamente para poder dar cuenta de los resultados de nuestro experimento, sino para poder dar cuenta de las potenciales interacciones entre ortografía y fonología en el procesamiento visual de palabras (véase Lupker, Acha, Davis \& Perea, en prensa, para un argumento similar con respecto a los efectos de los grafemas sobre el acceso léxico), así como para poder dar cuenta de las diferencias de procesamiento entre vocales y consonantes (Perea \& Acha, 2009; Vergara-Martínez, Perea, Marín \& Carreiras, 2011). Al respecto, la tarea de anagramas puede ser de utilidad en futuros trabajos para examinar los efectos de los grafemas (v.g., $\mathrm{CH}$ ), de las posibles diferencias entre la codificación posicional de vocales y consonantes o para estudiar más en detalle el papel que juega la posición del bigrama en la solución y en el anagrama.

Antes de finalizar, es importante indicar posibles ventajas e inconvenientes de la tarea de solución de anagramas. La principal ventaja es que permite estudiar la codificación de la información posicional utilizando un concepto de similitud diferente al usado hasta ahora, el concepto de poca semejanza entre dos cadenas. Cabe señalar que los resultados obtenidos replican los efectos obtenidos por otros estudios usando paradigmas clásicos como el priming enmascarado (v.g., Guerrera \& Forster, 2008) abriendo una nueva perspectiva para examinar cómo el sistema cognitivo codifica la posición de las letras en palabras. Una limitación de la tarea, sin embargo, es la gran variabilidad en las respuestas de los participantes. Ello es debido probablemente al prolongado tiempo de exposición de los estímulos (30 segundos), lo que ha limitado la potencia estadística de los análisis por ítem, en particular. No obstante, cabe señalar que si bien el tiempo máximo de exposición del anagrama era de 30 segundos, la mayor parte de los participantes (75\%) necesitó menos de 3 segundos para escribir la solución del anagrama. Dicha información es importante porque nos permitiría ajustar, en futuros trabajos con esta técnica, el tiempo de exposición del anagrama para así limitar la variabilidad de las respuestas y obtener tiempos más cercanos a los utilizados en tareas de decisión léxica, nombrado o igual-diferente. En todo caso, la réplica del clásico efecto de la frecuencia léxica confirma que ésta es una técnica apropiada para estudiar la organización de la información posicional en el léxico ortográfico, especialmente en secuencias altamente distorsionadas.

En resumen, los datos obtenidos en este trabajo muestran que la codificación de la posición de las letras es más apropiada en función de su contexto (posición relativa) que de forma independiente (posición absoluta). Además, este tipo de codificación está vinculada con una organiza- 
ción subléxica de tipo silábico. Ello implica la necesidad de cambios en los modelos de reconocimiento visual de palabras que pueden acomodar los efectos de transposición de letras.

\section{Referencias}

Acha, J. \& Perea, M. (2008). The effect of neighborhood frequency in reading: Evidence with transposed-letter neighbors. Cognition, 108, 290-300. doi:10.1016/j.cognition.2008.02.006.

Acha, J. \& Perea, M. (2010). Does kaniso activate CASINO? Input coding schemes and phonology in visual-word recognition. Experimental Psychology, 57, 245-251. doi:10.1027/1618-3169/a000029.

Álvarez, C., Carreiras M. \& de Vega, M. (2000). Syllable-frequency effect in visual word recognition: Evidence of sequential-type processing. Psicológica, 21, 341-374.

Álvarez, C., Carreiras, M. \& Perea, M. (2004). Are syllables phonological units in visual word recognition? Language and Cognitive Processes, 19, 427452. doi:10.1080/01690960344000242.

Álvarez, C., Carreiras, M. \& Taft, M. (2001). Syllables and morphemes: Contrasting frequency effects in Spanish. Journal of Experimental Psychology: Learning, Memory, and Cognition, 27, 545-555. doi:10.1037/02787393.27.2.545

Álvarez, C., de Vega, M. \& Carreiras, M. (1998). La sílaba como unidad de activación léxica en la lectura de palabras trisílabas. Psicothema, 10, 371386.

Ans, B., Carbonnel, S., \& Valdois, S. (1998). A connectionist multiple-trace model for polysyllabic word reading. Psychological Review, 105, 678-723. doi:10.1037/0033-295X.105.4.678-723.

Becker, C. A. (1976). Allocation of attention during visual word recognition. Journal of Experimental Psychology: Human Perception \& Performance, 2, 556566. doi:10.1037/0096-1523.2.4.556.

Becker, C. A. (1979). Semantic context and word frequency in visual word recognition. Journal of Experimental Psychology: Human Perception \& Performance, 5, 252-259. doi:10.1037/0096-1523.5.2.252.

Becker, C. A. (1985). What do we really know about semantic context effects during reading? In D. Besner, T. G. Waller \& G. E. MacKinnon (Eds.), Reading Research. Advances in theory and practice (Vol. 5, pp. 125-66). London: Academic Press.

Carreiras, M., Álvarez, C. \&. de Vega, M. (1993). Syllable frequency and visual word recognition in Spanish. Journal of Memory and Language, 32, 766780. doi:10.1006/jmla.1993.1038.

Carreiras, M. \& Perea, M. (2002). Masked priming effects with syllabic neighbors in the lexical decision task. Journal of Experimental Psychology: Human Perception and Performance, 28, 1228-1242. doi:10.1037/00961523.28.5.1228

Coltheart, M., Rastle, K., Perry, C., Langdon, R. \& Ziegler, J. (2001). A dual route cascaded model of visual word recognition and reading aloud. Psychological Review, 108, 204-256. doi:10.1037/0033-295X.108.1.204.

Conrad, M., Tamm, S., Carreiras, M. \& Jacobs, A. M. (2010). Simulating syllable frequency effects within an interactive activation framework. European Journal of Cognitive Psychology, 22, 861-893. doi:10.1080/09541440903356777.

Davis, C. J. (1999). Match calculator [computer software available at http://www.pc.rhul.ac.uk/staff/c.davis/Utilities/MatchCalc/index.htm ].

Davis, C. J. (1999). The self-organising lexical acquisition and recognition (SOLAR) model of visual word recognition. Dissertation Abstracts International: Section B: The Sciences and Engineering 62(1-B). Tesis doctoral no publicada, Universidad de New South Wales.

Davis, C. J. (2010). SOLAR versus SERIOL revisited. European Journal of Cognitive Psychology, 22, 695-724. doi:10.1080/09541440903155682.

Davis, C. J., \& Perea, M. (2005). BuscaPalabras: A program for deriving orthographic and phonological neighborhood statistics and other psycholinguistic indices in Spanish. Behavior Research Methods, 37, 665-671. doi:10.3758/BF03192738.

Ferrand, L., Grainger, J. \& Seguí, J. (1994). A study of masked form priming in picture and word naming. Memory \& Cognition, 22, 431-441.
Nota: La investigación realizada en este artículo ha sido parcialmente financiada por la Fundación Séneca de la Región de Murcia (05791/PPC/07) y por el Ministerio de Economía y Competitividad en España (PSI2011-26924).

doi:10.3758/BF03200868

Ferrand, L., Segui, J. \& Grainger, J. (1996). Masked priming of word and picture naming: The role of syllabic units. Journal of Memory and Language, 35, 708-723. doi:10.1006/jmla.1996.0037.

García-Orza, J., Perea, M., \& Muñoz, S. (2010). Are transposition effects specific to letters? Quarterly Journal of Experimental Psychology, 63, 1603-1618. doi:10.1080/17470210903474278.

Gómez, P., Ratcliff, R. \& Perea, M. (2008). The overlap model: A model of letter position coding. Psychological Review, 115, 577-601. doi: $10.1037 / \mathrm{a} 0012667$.

Grainger, I. J. \& Jacobs, A. M. (1996). Orthographic processing in visual word recognition: A multiple read-out model. Psychological Review, 103, 518-565. doi:10.1037/0033-295X.115.2.518.

Grainger, I. J., Granier, J. P., Farioli, F., Van Assche, E. \& Van Heuven, W. J. B. (2006). Letter position information and printed word perception: The relative-position priming constraint. Journal of Experimental Psychology: Human Perception and Performance, 32, 865-884. doi:10.1037/00961523.32.4.865.

Grainger, I. J. \& Van Heuven, W. J. B. (2003). Modeling Letter Position Coding in Printed Word Perception. En P. Bonin (Ed.), Mental lexicon: "Some words to talk about words" (pp. 1-23). Hauppauge, NY: Nova Science.

Guerrera, C. \& Forster, K. I. (2008). Masked form priming with extreme transposition. Language and Cognitive Processes, 23, 117-142. doi:10.1080/01690960701579722.

Hutzler, F., Bergmann, J., Conrad, M., Kronbichler, M., Stenneken, P. \& Jacobs, A. (2004). Inhibitory effects of first syllable frequency in lexical decision: an event related potential study. Neuroscience Letters, 372, 179184. doi:10.1016/j.neulet.2004.07.050.

Johnson, R. L. (2009). The Quiet Clam Is Quite Calm: Transposed-Letter Neighborhood Effects on Eye Movements During Reading. Journal of Experimental Psychology: Learning, Memory and Cognition, 35, 943-969. doi: $10.1037 / \mathrm{a} 0015572$.

Johnson, R. L., Perea, M. \& Rayner, K. (2007). Transposed letter effects in Reading: evidence from eye movements and parafoveal preview. Journal of Experimental Psychology: Human Perception and Performance, 33, 209-229. doi:10.1037/0096-1523.33.1.209.

Lee, C. H. \& Taft, M. (2009). Are onsets and codas important in processing letter position? A comparison of TL effects in English and Korean. Journal of Memory and Language, 60, 530-542. doi:10.1016/j.jml.2009.01.002.

Lee, C. H. \& Taft, M. (2011). Subsyllabic structure reflected in letter confusability effects in Korean word recognition. Psychonomic Bulletin and Review, 18, 129-134. doi:10.3758/s13423-010-0028-y.

Lupker, S. J., Acha, J., Davis, C. J., \& Perea, M. (en prensa). An investigation of the role of grapheme units in word recognition. Journal of Experimental Psychology: Human Perception and Performance.doi:10.1037/a0026886.

Lupker, S. J., Perea, M., \& Davis, C. J. (2008). Transposed letter priming effects: Consonants, vowels and letter frequency. Language and Cognitive Processes, 23, 93-116. doi:10.1080/01690960701579714.

Mayzner, M. S., \& Tresselt, M. E. (1962). Anagram solution times: A function of word transition probabilities. Journal of Experimental Psychology, 63, 510-513. doi:10.1037/h0043721.

McClelland, J. \& Rumelhart, D. (1981). An interactive activation model of context effects in letter perception: I. An account of basic findings. Psychological Review, 88, 375-407. doi:10.1037/0033-295X.88.5.375.

Muñoz, S., Perea, M., García-Orza, J, \& Barber, H. (2012). Electrophysiological signatures of masked transposition priming in a same-different task: Evidence with strings of letters vs. pseudoletters. Neuroscience Letters, 515, 71-76. doi:10.1016/j.neulet.2012.03.021.

Norris, D., Kinoshita, S. \& van Casteren, M. (2010). A stimulus sampling 
theory of letter identity and order. Journal of Memory and Language, 62 (3), 254-271. doi:10.1016/j.jml.2009.11.002.

Paap, K., Newsome, S. L., McDonald, J. E. \& Schvaneveldt, R. W. (1982). An activation-verification model for letter and word recognition: The word superiority effect. Psychological Review, 89, 573-594. doi:10.1037/0033-295X.89.5.573.

Perea, M., Abu Mallouh, R., \& Carreiras, M. (2010). The search of an input coding scheme: Transposed-letter priming in Arabic. Psychonomic Bulletin and Review, 17, 375-380. doi:10.3758/PBR.17.3.375.

Perea, M. \& Acha, J. (2009). Does letter position coding depend on consonant/vowel status? Evidence with the masked priming technique. Acta Psychologica, 130, 127-137. doi:10.1016/j.actpsy.2008.11.001.

Perea, M. \& Carreiras, M. (1998). Effects of syllable frequency and syllable neighborhood frequency in visual word recognition. Journal of Experimental Psychology: Human Perception and Performance, 24(1), 134-144. doi: 0.1037/0096-1523.24.1.134.

Perea, M. \& Carreiras, M. (2006a). Do transposed-letter similarity effects occur at a syllable level? Experimental Psychology, 53, 308-315. doi:10.1027/1618-3169.53.4.308.

Perea, M. \& Carreiras, M. (2006b). Do transposed-letter similarity effects occur at a prelexical phonological level? Quarterly Journal of Experimental Psychology, 59, 1600-1613. doi:10.1080/17470210500298880.

Perea, M. \& Carreiras, M. (2006c). Do transposed-letter effects occur across lexeme boundaries? Psychonomic Bulletin and Review, 13, 418-422. doi:10.3758/BF03193863.

Perea, M., \& Carreiras, M. (2008). Do orthotactics and phonology constrain the transposed-letter effect? Language and Cognitive Processes, 23, 69-92. doi:10.1080/01690960701578146.

Perea, M., Duñabeitia, J. A. \& Carreiras, M. (2008). Transposed-letter priming effects for close versus distant transpositions. Experimental Psychology, 55, 384-393. doi:10.1027/1618-3169.55.6.384

Perea, M. \& Estevez, A. (2008). Transposed letter similarity effects in naming pseudowords: Evidence from children and adults. European Journal of Cognitive Psychology, 20, 33-46. doi:10.1080/09541440701306941.

Perea, M. \& Lupker, S. J. (2003a). Transposed-letter confusability effects in masked form priming. In S. Kinoshita \& S. J. Lupker (Eds.). Masked priming: State of the art (pp. 97-120). Hove, UK: Psychology Press.

Perea, M. \& Lupker, S. J. (2003b). Does jugde activate COURT? Transposed-letter similarity effects in masked associative priming. Memory and Cognition, 31, 829-841. doi:10.1080/09541440701306941.

Perea, M. \& Lupker, S. J. (2004). Can CANISO activate CASINO? Transposed-letter similarity effects with nonadjacent letter positions. Journal of Memory and Language, 51(2), 231-246. doi:10.1016/j.jml.2004.05.005.

Perea, M., \& Lupker, S. J. (2007). La posición de las letras externas en el reconocimiento visual de palabras. Psicothema, 19, 559-564.

Perea, M., Nakatani, C., \& van Leeuwen, C. (2011). Transposition effects in reading Japanese Kana: Are they orthographic in nature? Memory and Cognition, 39, 700-707. doi:10.3758/s13421-010-0052-1.

Perea, M., \& Pérez, E. (2009). Beyond alphabetic orthographies: The role of form and phonology in transposition effects in Katakana. Language and Cognitive Processes, 24, 67-88. doi:10.1080/01690960802053924.

Perea, M., Winskel, H., \& Ratitamkul, T. (2012). On the flexibility of letter position coding during lexical processing: The case of Thai. Experimental Psychology,59, 68-73. doi:10.1027/1618-3169/a000127.

Prinzmetal, W., Treiman, R. \& Rho, S. H. (1986). How to see a reading unit. Journal of Memory and Language, 25, 461-475. doi:10.1016/0749596X(86)90038-0.

Quintanilla, P. (2006). El papel de la sílaba en el reconocimiento visual de palabras. Tesis doctoral no publicada. Universidad de Murcia, Departamento de Psicología Básica y Metodología.

Rayner, K., White, S. J., Johnson, R. L. \& Liversedge, S. P. (2006). Raeding Wrods with jumbled lettres. There is a cost. Psychological science, 17, 192193. doi:10.1111/j.1467-9280.2006.01684.x.

Schoonbaert, S. \& Grainger, J. (2004). Letter position coding in printed word perception: Effects of repeated and transposed letters. Language and Cognitive Processes, 19, 333-367. doi:10.1080/01690960344000198.

Schwartz, R. (1968). Anagram solving: A function of the frequency of vowel-consonant pattern. Psychonomic Science, 13, 229-230.

Sebastián, N., Marti, M. A., Cuetos, F. \& Carreiras, M. (2000). LEXESP. Léxico informatizado del español. Barcelona ediciones de la Universidad de Barcelona.

Sternberg, S. (1969). The discovery of processing stages: Extensions of Donders' method. In W.G. Koster (Ed.), Attention and performance II (pp. 276-315). Amsterdam: North-Holland

Topper, G. E., Macey, W. H., \& Solso, R. L. (1973). Bigram versatility and bigram frequency. Behavior Research Methods, 5(1), 51-53. doi:10.3758/BF03200121

Tresselt, M. E. \& Mayzner, M.S. (1968). Anagram solution times: A function of single and double letters solutions words. Journal of Verbal Learning and Verbal Behaviour, 7, 128-132. doi:10.1016/S0022-5371(68)80176-8.

Velan, H. \& Frost, R. (2011). Words with and without internal structure: What determines the nature of orthographic and morphological processing? Cognition, 118, 141-156. doi:10.1016/j.cognition.2010.11.013.

Velandrino, A. P. (1985). La tarea de identificación de anagramas y el proceso de codificación. Anales de Psicología, 2, 129-143.

Vergara-Martínez, M., Perea, M., Marín, A., \& Carreiras, M. (2011). The processing of consonants and vowels during letter identity and letter position assignment in visual-word recognition: An ERP study. Brain and Language, 118, 105-117. doi:10.1016/j.bandl.2010.09.006.

Whitney, C. (2001). How the brain encodes the order of letters in a printed word: The SERIOL model and selective literature review. Psychonomic Bulletin and Review, 8, 221-243. doi:10.3758/BF03196158.

Whitney, C. \& Cornelissen, P. (2005). Letter position encoding and dyslexia. Journal of Research in Reading, 28, 274-301. doi:10.1111/j.14679817.2005.00270.x

(Articulo recibido: 9-12-2011, revisión: 19-4-2012, aceptado: 2-5-2012) 